\title{
The chemistry of carbon dust formation
}

\author{
Isabelle Cherchneff \& Piero Cau \\ Department of Physics, UMIST, PO Box 88, Manchester M60 1QD, \\ United Kingdom
}

\begin{abstract}
.
We shall review the various types of chemistry involved in the formation of carbonaceous material present in carbon-rich AGB stars, mainly amorphous carbon, silicon carbide and other metal carbides discovered in pre-solar stardust extracted from meteorites. The chemistry is discussed in the context of laboratory experiments and their application to circumstellar AGB winds. Emphasis is put on polycyclic aromatic hydrocarbons (PAHs), titanium carbide clusters and silicon carbide grains. Attempt to explain the condensation sequences derived from the study of pre-solar grains of meteoretical origin is made on the basis of physio-chemical modeis which describe the periodically shocked gas close to the photosphere of AGB stars.
\end{abstract}

\section{Introduction}

Carbon-rich stars ascending the Asymptotic Giant Branch (AGB) are known to form copious amount of dust at the base of their wind, as shown by the strong infrared (IR) excess they emit and the large mass-loss rate they develop. Except for the band seen at $\sim 11.3$ microns in emission or absorption in most carbon stars and ascribed to silicon carbide ( $\mathrm{SiC}$ ) grains, the IR thermal emission of dust is usually featureless and can be well reproduced by a distribution of amorphous carbon (AC) particles (Groenewegen 1997). A new source of information on stardust comes from the study of pre-solar grains extracted from meteorites (Bernatowicz et al. 1991, 1996). These studies bring evidence for various sequences of nucleation and condensation in carbon stars, in particular the presence of transition metal carbides such as titanium or zirconium carbides embedded in the aromatic lattice, and the absence of silicon carbide clusters in graphite pre-solar grains.

Many studies have investigated the condensation of carbon grains in stationary or time-dependent winds (Gail \& Sedlmayr 1988; Fleischer et al. 1994; Höfner et al. 1995) in order to explain ways of generating outflows and predict mass-loss rates. However, the nucleation and condensation of dust grains, which involve a full description of the chemistry describing the passage of the gas to the solid phase is not considered. Furthermore, the dust formation zone in AGB stars is characterised by the passage of strong periodic shocks induced by stellar pulsation implying physical conditions far from equilibrium. Therefore, it 
is important to bear in mind that studies of dust formation should consider a non-equibrium chemistry for the description of dust nucleation.

In this review, we discuss the various types of chemistry involved in the formation of $\mathrm{AC}$ grains, transition metal carbides and $\mathrm{SiC}$ clusters in the laboratory and the possible relevance of these mechanisms to stellar environments. We also present preliminary results on the formation of polycyclic aromatic hydrocarbons (PAHs) and molecular precursors to $\mathrm{TiC}$ clusters in the inner shocked wind of the carbon star IRC +10216 and draw potential explanations to the condensation sequences derived from meteoretical studies.

\section{The chemistry of carbon grain formation in the laboratory}

\subsection{Amorphous Carbon}

As already mentioned and investigated by several authors (Frenklach \& Feigelson 1989; Frenklach et al. 1989; Cherchneff et al. 1992; Cadwell et al. 1994; Cherchneff et al. 1998), the chemical composition of the photospheres of carbon stars and some of the physical parameters characterising the inner stellar winds are very similar to conditions encountered in the combustion and/or the infrared pyrolysis of hydrocarbons in the laboratory. Indeed, acetylene, $\mathrm{C}_{2} \mathrm{H}_{2}$, is the dominant carbon-bearing molecule after carbon monoxide in the photospheres of carbon stars which have typical temperatures are $2500 \mathrm{~K}$. Such temperatures are also found in flames rich in acetylene which are efficient sources of amorphous carbon particles in the form of soot. Therefore, there exists a natural link between the two environments which permits the use of combustion chemistry to model the formation of AC grains in stars.

The major steps of soot particle nucleation and condensation are illustrated in Fig. 1. The first step referred to as nucleation consists of forming large aromatic molecules from the acetylene-rich gas phase. The second step is the condensation stage where a three-dimensional, small solid particle forms from large aromatic precursors. Formation of the first aromatic ring involves the isomerisation of acetylene and the formation of larger hydrocarbons via ter-molecular and bi-molecular reactions which may have relatively high activation energies. However, these are overcome due to the high gas temperature in the flame, shock-tube or stellar environment. The nucleation step includes the closure of the first aromatic ring ( $\equiv$ benzene, $\mathrm{C}_{6} \mathrm{H}_{6}$ ) and the subsequent growth of PAHs. Once benzene or phenyl are formed, the growth to PAHs can be simply represented by a set of chemical reactions which consists of (Frenklach \& Feigelson 1989; Cherchneff et al. 1992; Frenklach \& Feigelson 1997):

\section{Acetylene addition to aromatic radical}

2. Atomic hydrogen extraction from the aromatic ring to form a radical site

3. Second acetylene addition and closure of the ring.

Because of the high gas densities, the reverse reactions for all processes have to be considered. This series of reactions occurs for a certain temperature window determined by the local gas number density and ring growth is favoured 


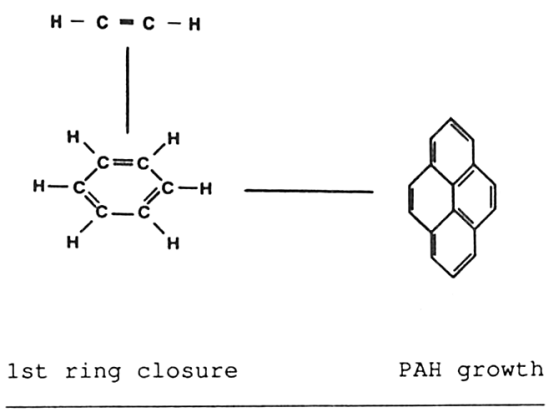

NUCLEATION

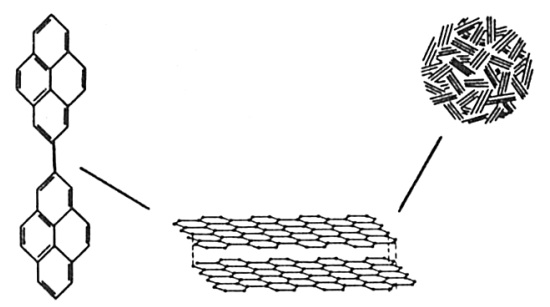

Coagulation Platelet soot particle

CONDENSATION

Figure 1. Dominant pathways to PAH growth (adapted from Cherchneff et al. 1992).

when Reaction 2 is at equilibrium but the $\mathrm{C}_{2} \mathrm{H}_{2}$ addition in Reaction 3 becomes irreversible. In flames, this temperature window is $900-1100 \mathrm{~K}$, but in astrophysical environments, in particular in the inner shocked regions of carbon star winds, this window can vary greatly as shown in Sect. 3 .

As for condensation, the various stages comprise a series of chemical processes which permit the growth of small solid condensates. Whereas nucleation treats the conversion of linear gas-phase species into planar, large aromatic molecules, condensation involves the growth of planar species into threedimensional solid grains. Condensation is initiated with the formation of PAH dimers where the aromatics are linked by van der Waals forces. Condensation products form simultaneously and these differ from the dimers by the formation of a six-membered or five-membered ring as a link between the two aromatic rings. For example, both dimers and condensation products of pyrene are observed in high-temperature pyrolysis of pyrene and the growth to soot particles was suggested to take place through aromatic polymerisation and cyclodehydrogenation rather than through acetylene addition (Mukherjee et al. 1994). The growth then proceeds through coagulation of condensation products of PAH molecules, deposition of carbon via acetylene surface reactions and surface condensation of free PAH molecules on the grains.

\subsection{Metal carbides}

The reactions of early transition metals with hydrocarbons and the resulting formation of various metal-carbon clusters have been extensively studied in laser-induced plasma reactor experiments (Guo et al. 1992; Wei et al. 1992). The metal usually considered were titanium, vanadium and zirconium and the metal-carbon clusters observed spanned many molecular weights in the mass spectrometer. An important discovery was the identification of a dominant 


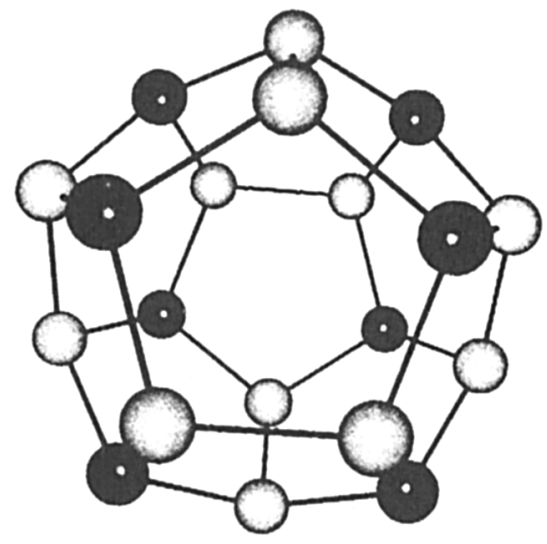

Figure 2. A possible $\mathrm{T}_{\mathrm{h}}$ point group structure for $\mathrm{Ti}_{8} \mathrm{C}_{12}$ (from Guo et al. 1992).

cluster among the cluster distribution peaking at 528 atomic mass units and due the metallo-carbohydrene $\mathrm{M}_{8} \mathrm{C}_{12}$ (or met-car), where $\mathrm{M}$ is the transition metal under consideration. Due to its 'super-magic' nature and the fact that the cluster was always observed dominant whatever the hydrocarbon reactant used (methane, $\mathrm{CH}_{4}$, ethylene, $\mathrm{C}_{2} \mathrm{H}_{4}$, and acetylene), it was suggested that the species had a cage structure to explain its stability and ubiquity (Guo et al. 1992; Reddy \& Khanna 1993; Wei \& Castleman 1994; Cartier et al. 1996). An idealised pentagonal dedocadedron structure for $\mathrm{Ti}_{8} \mathrm{C}_{12}$ is illustrated in Fig. 2 and shows that each titanium atom can bond to three carbon atoms through $\mathrm{Ti}-\mathrm{C} \sigma$-bonds while each carbon atom is bonded to two titanium atoms and its adjacent carbon through a $\mathrm{C}-\mathrm{C} \sigma$-bond.

The chemical mechanisms leading to the formation of 'met-car' clusters have not been identified yet but there is evidence from the cluster size distribution for structural growth through addition of the $\mathrm{MC}_{2}$ unit (Guo et al. 1992) and closure at $\mathrm{M}_{8} \mathrm{C}_{12}$. The chemistry of met-car molecular precursors is still unexplored but studies on organometallics and the gas-phase reactions of transition metals with various hydrocarbons have shown that bare transition metal cations $\left(\mathrm{Ti}^{+}, \mathrm{Zr}^{+}\right.$, $\mathrm{V}^{+}$, etc.) are very reactive chemicals that can break $\mathrm{C}-\mathrm{H}$ and $\mathrm{C}-\mathrm{C}$ bonds in alkanes and alkenes (Byrd et al. 1982; Tolbert \& Beauchamp 1986). A typical reaction of $\mathrm{Ti}^{+}$with $\mathrm{C}_{2} \mathrm{H}_{4}$ gives

$$
\mathrm{Ti}^{+}+\mathrm{C}_{2} \mathrm{H}_{4} \rightarrow \mathrm{TiC}_{2} \mathrm{H}_{2}+\mathrm{H}_{2}
$$

where two hydrogen atoms have been eliminated to form $\mathrm{H}_{2}$.

The reactions of neutral transition metals with hydrocarbons have been investigated with less extent. Ritter \& Weisshaar (1990) and Ritter et al. (1992) 
have studied the kinetics of neutral transition metals in the gas phase and conclude that they are less reactive than their cation counterparts. In particular, they derive a low reaction rate for the bimolecular reaction between neutral titanium (and vanadium) with $\mathrm{C}_{2} \mathrm{H}_{4}$. The reaction of neutral titanium with acetylene remains unexplored at present time, although it is known from the study of Guo et al. (1992) that $\mathrm{Ti}^{+}$(and possibly neutral $\mathrm{Ti}$ ) must react very efficiently with acetylene to explain the very large abundance of $\mathrm{Ti}_{8} \mathrm{C}_{12}$ observed.

\subsection{Silicon carbide}

Silicon carbide powders have been extensively studied in the laboratory as a result of the pyrolysis of silane, $\mathrm{SiH}_{4}$, and various hydrocarbons (Harano et al. 1990; Lirhmann \& Cauchetier 1994). Although the end products and the powder are usually well characterized, the mechanisms explaining the formation of $\mathrm{SiC}$ cluster precursors and the condensation processes are still unclear. Fantoni et al. (1991) have investigated the by-products of $\mathrm{SiC}$ cluster formation and have identified with the help of infrared spectroscopy the presence of $\mathrm{C}_{2}$ and $\mathrm{SiC}_{2}$ as intermediates in the nucleation and condensation processes. Harano et al. (1990) in their laser-induced plasma experiment have brought evidence for the presence of several $\mathrm{Si}$ and $\mathrm{SiC}$ clusters in the final cluster distribution. In particular they found that $\mathrm{SiC}_{n}, \mathrm{Si}_{2} \mathrm{C}_{n}$ (with $\mathrm{n}>1$ ) and pure carbon clusters were dominant as the percentage of hydrocarbons was raised in the buffer gas. There is no 'super-magic' molecule such as met-cars in the cluster distribution indicating that no specific cage structure can act as condensation nucleus in the grain growth processes. This result may hint to the fact that $\mathrm{SiC}$ and metal carbides have very different fates in stellar winds, the former acting as a separate dust population whereas the latter is trapped in the aromatic lattice of the forming grain.

\section{Condensation sequences and preliminary models}

An important implication of the studies of meteorites and the extraction of isotopically anomalous pre-solar graphite spherules, which origin has been ascribed to carbon stars on the AGB, is the possibility to gain insight on dust condensation processes and to test theoretical models of grain formation. For example, the study of graphite spherules by Bernatowicz et al. (1996) points to various possible dust condensation sequences in carbon stars, as illustrated in Table 1.

Transmission electron microscopy and electron diffraction studies conducted by Bernatowicz et al. have provided evidence for the presence of three types of configuration for the spherules: (1) a core of small graphene sheets (size 40 $\AA$ ) and small PAHs (size $<10 \AA$ ) surrounded by a well graphitized carbon rim. About $80 \%$ of the spherules are of this type; (2) a core of metal carbide nanocrystals surrounded by the same rim as in (1); (3) metal carbide nanocrystals randomly distributed in the graphite spherule. Such information can help understanding the condensation of AC grains in carbon stars, in particular, it strengthens the fact that silicon carbide clusters are not observed to act as condensation nuclei to AC particles as it has been proposed by Frenklach et al. (1989) and Cadwell et al. (1994). 
Table 1. Condensation sequences observed in pre-solar graphite spherules (from Cherchneff 1998).

\begin{tabular}{lllll}
\hline \hline Core & Mantle & $\begin{array}{l}\text { Core size } \\
(\AA)\end{array}$ & $\begin{array}{l}\text { Graphite rim } \\
\text { size }(\mu \mathrm{m})\end{array}$ \\
\hline 1 & Graphene + PAHs & Graphitic concentric sheets (GCS) & $?$ & $0.1-0.4$ \\
2 & TiC, ZrC, MoC & GCSs & $50-300$ & $0.1-0.4$ \\
3 & none & metal carbides spread in GCSs & - & $0.1-0.4$ \\
\hline
\end{tabular}

In order to gain insight on condensation sequences in a mixture of dust grains, we have investigated the nucleation and early condensation of carbon grains using a formalism based on combustion chemistry simultaneously to the formation of molecular precursors to titanium carbide clusters, in particular the molecule $\mathrm{TiC}_{2}$. We apply the chemistry to the dust formation region close to the stellar photosphere of the archetypical carbon star IRC +10216 and use the model for the inner, periodically shocked wind as described in Willacy \& Cherchneff (1998) and Cherchneff et al. (1998). For AC grain precursors, we consider the formation of free PAHs up to coronene $\left(\mathrm{C}_{24} \mathrm{H}_{12}\right)$ and their dimers. We do not include destruction processes for the latter as we assume that the dimers convert quickly to condensation products in which the two aromatic molecules are bridged by a pentagonal or an aromatic ring. These products are very stable and are observed to form at the expense of PAH dimers in aromatic pyrolysis experiments at high temperature (Mukherjee et al. 1994).

We define a PAH (dimer) yield as the ratio between the number of carbon atoms locked in PAHs (dimers) divided by the number of carbon atoms initially present in hydrocarbons (Cherchneff et al. 1992). The yields for benzene, PAHs and aromatic dimers are presented in Fig. 3 as a function of stellar radius. The yields at each radius correspond to the values calculated in the periodic shocks and in the gas excursions induced by the shocks over two pulsation periods (see Cherchneff et al. 1998, for more details). From inspection of Fig. 3, several conclusions can be drawn. First, the non-equilibrium chemistry favours the formation of aromatic species close to the photosphere and the shock formation radius (equal to $1.2 \mathrm{R}_{\star}$ in this model). Benzene starts to form as early as $1.4 R_{\star}$ and represents the dominant aromatic hydrocarbon until $1.7 R_{\star}$. At this radius, the conversion of single-ring aromatics to PAHs starts as the growth due to acetylene addition becomes irreversible, but it is important to notice that the temperature at which this growth starts is $\sim 1700 \mathrm{~K}$ for the physical parameters of the gas excursion considered (typical gas number density of $\sim 10^{13}-10^{14} \mathrm{~cm}^{-3}$ ). This temperature is much higher than the temperature window derived by Frenklach \& Feigelson (1989) and Cherchneff et al. (1992) as these previous studies considered stationary expanding outflows and lower gas number densities. PAH dimers form in large amount along with the growth of their aromatic progenitors and yields between $10^{-5}$ and $10^{-4}$ are obtained.

The conversion of atomic $\mathrm{Ti}$ to $\mathrm{TiC}_{2}$ is illustrated in Fig. 4 along with the $\mathrm{PAH}$ formation yield. We have modelled the formation of $\mathrm{TiC}_{2}$ assuming that 


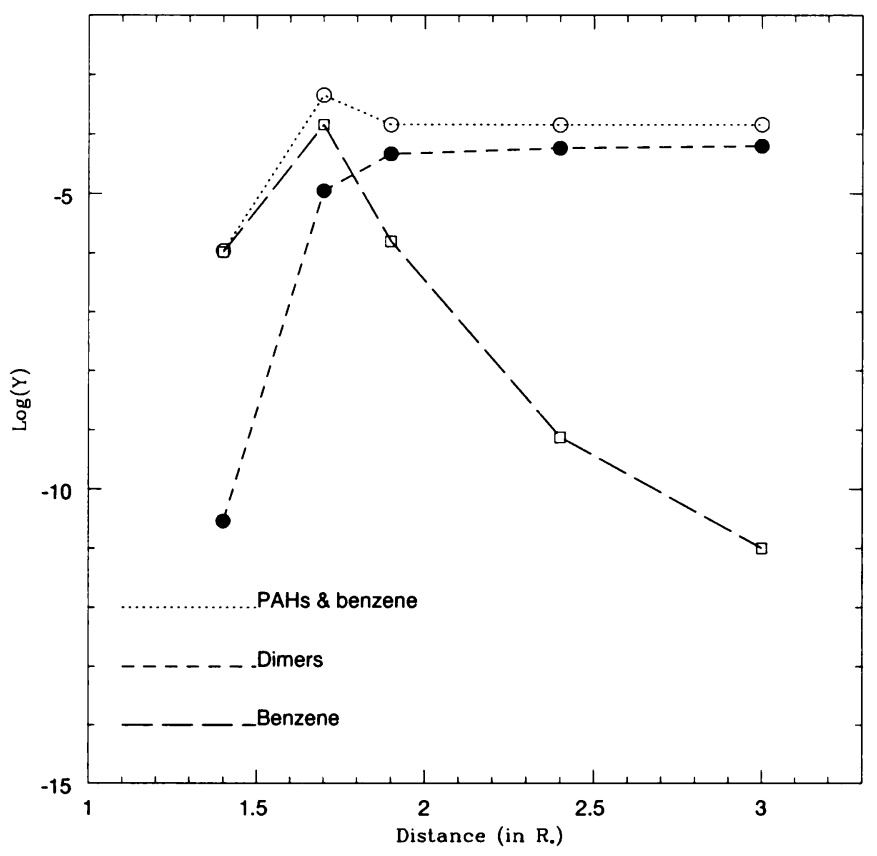

Figure 3. Benzene, PAH and PAH dimer formation yields as a function of stellar radius in the inner wind of IRC +10216 .

atomic titanium reacts with acetylene and using the dehydrogenation property of neutral Ti. Considering the reaction

$$
\mathrm{Ti}+\mathrm{C}_{2} \mathrm{H}_{2} \rightarrow \mathrm{TiC}_{2}+\mathrm{H}_{2}
$$

we use a very low rate $k=10^{-16} \mathrm{~cm}^{3} \mathrm{~s}^{-1}$ as suggested by Ritter et al. (1992). It appears that all the phostospheric $\mathrm{Ti}$ can be converted very rapidly to $\mathrm{TiC}_{2}$ in the first shock and its excursion and that the process is irreversible once this transformation takes place. This implies that precursors to TiC clusters can form at very high temperatures, and certainly before PAH growth.

\section{Discussion}

The formation of AC grains in carbon stars is far from beeing understood yet but a description of the various steps involved in the passage from gas to solid phase relying on non-equilibrium chemistry appears to give promising results. A first important point is that PAHs and their products form at higher gas temperatures and densities than previously thought resulting in more efficient aromatic growth. Further growth processes such as acetylene deposition and 


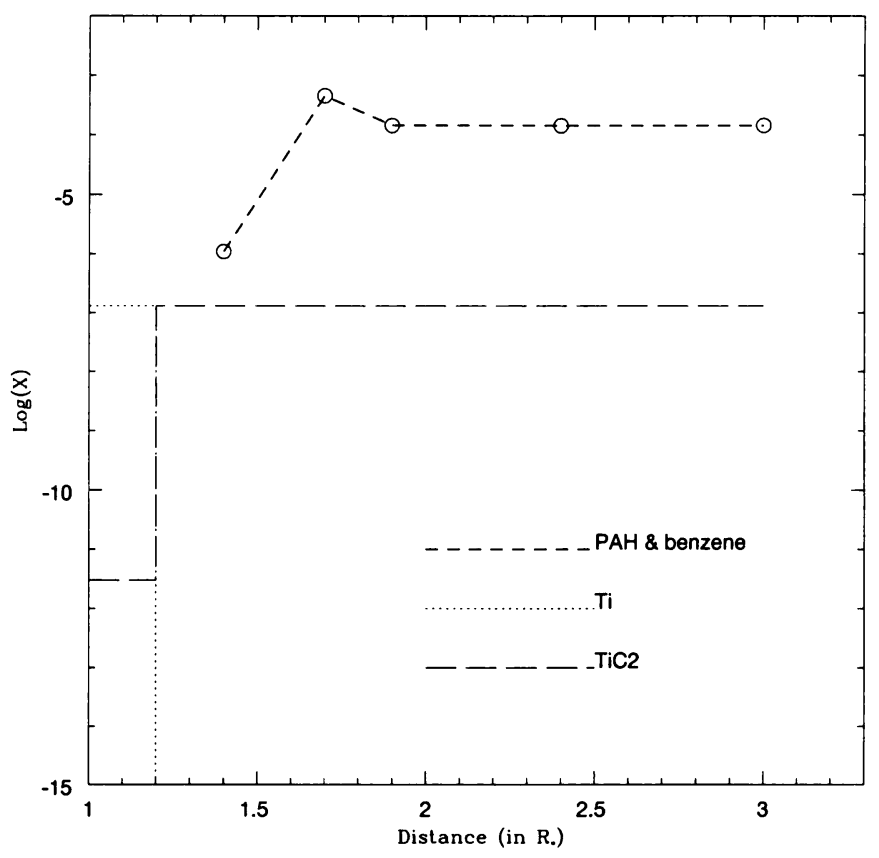

Figure 4. The conversion of atomic Titanium to $\mathrm{TiC}_{2}$ along with the $\mathrm{PAH}$ formation yield as a function of stellar radius in the inner wind of IRC +10216 .

condensation product coalescence must be investigated for the new conditions encountered in the inner wind.

Although there is evidence for formation of $\mathrm{TiC}$ precursors at high temperatures and certainly before the growth of aromatics, the conversion of $\mathrm{TiC}_{2}$ into met-car molecules and larger clusters remains an open question. If this conversion is fast enough and occurs efficiently at high temperatures, then TiC clusters are expected to form before the aromatics resulting in a core-mantle structure for carbon grains as seen in some pre-solar grains. This scenario could occur in stars in which strong shocks develop or characterised by high effective temperature. In case the conversion to larger $\mathrm{TiC}$ clusters is slow, their formation would be delayed to the regions of aromatic growth, resulting in a mixed AC grain where $\mathrm{TiC}$ cristals are spread in the lattice. This scenario could also happen for the slower shocks or cooler stars. As for the pre-solar grains containing only an $\mathrm{AC} / \mathrm{PAH}$ core, can they correspond to inhibited $\mathrm{TiC}$ cluster formation or to a case where $\mathrm{Ti}_{8} \mathrm{C}_{12}$ clusters form but do not grow, being below the resolution limit to be detected but still acting as small condensation nuclei to AC dust grains? 
The case of silicon carbide is even more puzzling and much less well defined as there exist only a few measured rates for reactions involving silicon compounds. The fact that no 'super-magic' cluster as the met-car molecule has been detected as bi-products of $\mathrm{SiC}$ powder formation could restrict the efficiency of $\mathrm{SiC}$ formation in stellar environments. If the chemistry of $\mathrm{SiC}$ grain growth is slower than that of transition metal carbides and aromatics, the $\mathrm{SiC}$ dust formation will certainly be decoupled from other dust condensation mechanisms, resulting in a separate grain population. This question can only be answered by the combined efforts of the various scientific communities working on carbon dust in the laboratory and in space.

\section{References}

Bernatowicz T.J., Amari S., Zinner E.K., Lewis R.S., 1991, ApJ 373, L73

Bernatowicz T.J., Cowsik R., Gibbons P.C., et al., 1996, ApJ 472, 760

Byrd G.D., Burnier R.C., Freiser B.S., 1982, J. Am. Chem. Soc. 104, 3565

Cadwell B.J., Wang H., Feigelson E.D., Frenklach M., 1994, ApJ 429, 285

Cartier S.F., May B.D., Castleman A.W., 1996, J. Phys. Chem. 100, 8175

Cherchneff I., 1998, in "Abundance profiles: Diagnostic tools for Galaxy history", San Francisco, ASP Conf. Ser. 147, p. 179

Cherchneff I., Cau P., Tielens A.G.G.M., 1998, A\&A, submitted

Cherchneff I., Barker J.R., Tielens A.G.G.M., 1992, ApJ 401, 269

Fantoni R., Bijen F., Djurić N., Piccirillo S., 1991, Appl. Phys. B 52, 176

Fleischer A.J., Gauger A., Sedlmayr E., 1994, A\&A 297, 543

Frenklach M., Carmer C.S., Feigelson E.D., 1989, Nature 339, 196

Frenklach M., Feigelson E.D., 1989, ApJ 341, 372

Frenklach M., Feigelson E.D., 1997, in "From Stardust to Planetesimals", Y. Pendleton (ed.), ASP Conference Series 122, p. 107

Gail H.-P., Sedlmayr E., 1988, A\&A 206, 153

Groenewegen M.A.T., 1997, A\&A 317, 503

Guo B.C., Kerns K.P., Castleman A.W., 1992, Science 255, 1411

Harano A., Kinoshita J., Koda, Seiichiro, 1990, Chem. Phys. Letters 172, 219

Höfner S., Feuchtinger M.U., Dorfi E.A., 1995, A\&A 297, 815

Lihrmann J.-M., Cauchetier M., 1994, J. European Ceramic Soc. 13, 41

Mukherjee J., Sarofim A., Longwell J.P., 1994, Combustion and Flame 96, 191

Reddy B.V., Khanna S.N., 1993, Chem. Phys. Letters 209, 104

Ritter D., Weissharr J.C., 1990, J. Am. Chem. Soc. 112, 6425

Ritter D., Carroll J.J., Weissharr J.C., 1992, J. Phys. Chem. 96, 10636

Tolbert M.A., Beauchamp J.L., 1986, J. Am. Chem. Soc. 108, 7509

Wei S., Guo B.C., Purnell J., Buzza S., Castleman A.W., 1992, J. Phys. Chem. 96,4166

Wei S., Castleman A.W., 1994, Chem. Phys. Letters 227, 305

Willacy K., Cherchneff I., 1998, A\&A 330, 676 


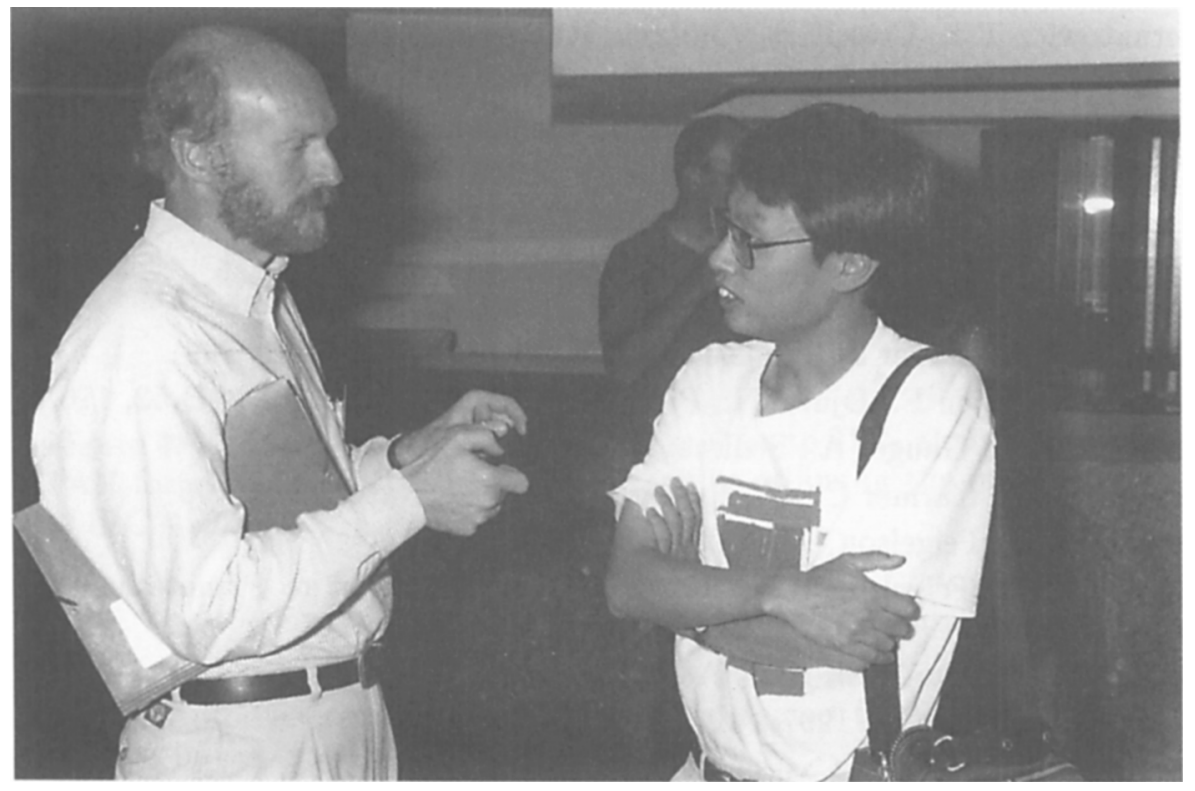

Hans Olofsson and Issei Yamamura 